\title{
Messina Earthquake, 1908
}

\author{
Roberto Sciarrone \\ Università di Roma La Sapienza \\ roberto.sciarrone@uniroma1.it
}

\section{Doi:10.5901/ajis.2013.v2n2p317}

\begin{abstract}
There are many stories, testimonials, articles and books about what was considered one of the greatest disasters in human history. An entire city, rich in history and traditions, was erased forever by the destructive fury of nature. In 37 seconds more than two thousand years of culture and pride disappeared together with the awareness of being a dynamic, historically ancient and rich diverse cultural influences over the centuries embellished Sicily. The social capital of the city crumbled because of the large number of deaths, an entire modus operandi vanished beneath the rubble of the baroque buildings, swept away by waves and finished apocalyptic fires that came copiousone after another. Messina, for many, died that day, but during the days following the great disaster its dynamic and eclectic spirit, which resulted from a story marked by multiculturalism, provided a test of absolute decisiveness.
\end{abstract}

Keywords: Messina, Earthquake, Strait of Messina, Tsunami, Italian Army, Russian Navy

\section{Preamble}

Alle ore 5:21' del 28 dicembre 1908, un violento terremoto del 10. grado della scala Mercalli distrusse le città di Messina e Reggio Calabria e numerosi villaggi e paesi vicini. II sisma, della durata di trentasette secondi, ebbe il suo epicentro nello Stretto, la scossa, prima a carattere sussultorio e quindi ondulatorio, fu seguita a distanza di due minuti da tre ondate di maremoto che completarono la distruzione delle zone ripariali, penetrando sulla terra per oltre cento metri e modificando sensibilmente il fondo marino. Secondo stime dell'epoca le vittime furono oltre centomila, anche se i morti di cui si richiese la registrazione furono 30.000 a Messina e 8.900 a Reggio Calabria. Assieme alla distruzione materiale della città, il terremoto cancellò la società messinese, le tradizioni e la storia di tremila anni di civiltà. Messina e il mare, un binomio che racchiude le più belle pagine di storia della città siciliana, ma anche le più drammatiche. Situata sullo stretto omonimo, Messina fu fondata nel sec. VIII a.C. dai coloni greci calcidesi, ebbe il nome di Zancle, che significa falce, poiché si estendeva in un'insenatura di forma semicircolare (Consolo, 2005). Invasa dai messeni nel V secolo, assunse poi il nome di Messanion, trasformato nella forma latina Messana. Durante i conflitti tra greci e cartaginesi passò dall'uno all'altro dei contendenti, finché nel 241 a.C. se ne impadronirono i romani. In epoca bizantina Messina ebbe una certa libertà e i suoi traffici prosperarono fino a quando, durante l'anno 843, fu conquistata dai saraceni che dopo due secoli si arresero ai normanni. Durante il Medioevo la città ricoprì un ruolo di grande importanza: il suo porto fu il punto di partenza delle crociate cristiane verso la Terrasanta, in quel periodo Messina iniziò a sviluppare una rete commerciale e un'attività economica di primaria importanza. Nel Cinquecento il porto di Messina era costantemente frequentato da navi che giungevano dal Mediterraneo al Mar del Nord, eletta finanche punto d'incontro della coalizione cristiana guidata da don Giovanni d'Austria contro quella ottomana di Mehmet Alì Pascià nella sanguinosa battaglia di Lepanto (Motta, 1998). Della vitalità commerciale e culturale della città dello Stretto in quell'epoca Giovanna Motta ne esalta i particolari: "Il Cinquecento era stato senza dubbio il secolo della gloria di Messina lungo il quale avevano lasciato nobili tracce le splendide opere del fiorentino Montorsoli, la lanterna e la fontana del Nettuno, e quelle del carrarese Andrea Calamech, autore della statua di don Giovanni d'Austria e del Grande Ospedale" (Motta, 2008). Lo sviluppo del capitalismo in area fiamminga e inglese determinò, poi, lo spostamento dei traffici economici verso l'Europa del nord a svantaggio del Mediterraneo, ciò causò una minore incidenza nel commercio internazionale di Messina. Tuttavia nel XVII secolo la città ridisegnò la sua immagine, fu costruita la "palazzata", serie di palazzi affacciati sul mare, furono eretti chiese e conventi, fortificò le zone portuali e organizzò al meglio le strutture militari e di soccorso (Frisina, 2008). La rivolta antispagnola del 1674, persa dai messinesi, rappresentò forse l'ultima occasione di cambiamento per la città e ne determinò la fine dei privilegi ottenuti nei secoli precedenti (Ribot, 2011). La peste del 1743 e il terremoto del 1783 acuirono il processo di decadenza iniziato un secolo prima, durante i primi anni dell'Ottocento si ebbe un ritorno alle origini grazie agli inglesi, in 
funzione antinapoleonica, ma ormai la città dello Stretto aveva perduto da qualche tempo la sua rilevanza portuale internazionale. Era questa la cornice storica, economica e sociale con cui la città dello Stretto si presentava all'alba del 28 dicembre 1908.

\title{
2. I particolari del terrore e i primi soccorsi: le navi russe
}

Sono numerosi i racconti, le testimonianze, gli articoli e i libri riguardo quello che fu considerato uno dei più grandi disastri della storia dell'umanità. Un'intera città, ricca di storia e tradizioni, fu cancellata per sempre dalla furia distruttrice della natura. In trentasette secondi sparirono più di duemila anni di cultura, sparì la messinesità, l'orgoglio e la consapevolezza di essere una società dinamica, storicamente antica e ricca di diverse contaminazioni culturali che nei secoli impreziosirono la Sicilia. II tessuto sociale che la componeva si sfilacciò a causa dei numerosissimi morti, un intero modus operandi svanì sotto le macerie dei palazzi barocchi, travolto da onde apocalittiche e finito dagli incendi susseguitisi copiosi. Messina, per molti, morì quel giorno, ma il suo spirito dinamico ed eclettico, frutto di una storia volta al multiculturalismo, fornì una prova di assoluta risolutezza durante i giorni seguenti il grande disastro. Ciò che i sopravvissuti non dimenticheranno mai sono i volti dei marinai russi, scolpiti tra i detriti, tra le fiamme e i corpi che galleggiavano in mare, lì dove un tempo regnava il commercio di uno dei più importanti porti del Mediterraneo. Numerose, al riguardo, le testimonianze che i superstiti, i giornalisti, gli scrittori dell'epoca ci hanno lasciato circa le gesta dei marinai russi, giovani ragazzi che per primi portarono aiuto alla città di Messina, e che rimarranno per sempre un capitolo, seppur doloroso, della storia cittadina. Prima di analizzare le varie testimonianze sull'eroismo che i marinai russi dimostrarono è d'obbligo riportare una delle più strazianti descrizioni dell'epoca, scritta dal prof. Arnaldo Bruschettini dell'università di Messina, il quale abitava in un palazzo tra i pochissimi rimasti in piedi, in pieno centro in via Garibaldi, riportata dal "Mattino" il 31 dicembre 1908:

\begin{abstract}
Messina è un ammasso di rovine sulle quali continua a divampare l'incendio. I superstiti sono poche migliaia. Lungo la spiaggia devastata, i sopravvissuti urlavano pazzi di terrore, tutti seminudi. Avvicinandosi alla Marina, si scorgeva la famosa Palazzata, sino al Municipio, completamente distrutta. Mancavamo di viveri, di luce e di acqua, scene terrorizzanti si svolgevano tutte intorno, cagionate dalla gente che andava alla requisizione dei viveri con le rivoltelle in pugno. Mancavano i soldati per reprimere le violenze, perché essi erano periti, sotto le macerie delle caserme crollate. Mi recai all'Osservatorio. Al cader della notte, lo spettacolo era ancor più terrorizzante e lugubre, per il divampare degli incendi in numerosi punti della città. A un certo punto, la notte oscura fu ravvivata dalla luce di parecchi riflettori elettrici, proiettata dal mare, erano i ferry boats. Passammo la notte tra scosse paurose. All'alba, vedemmo, tra il fumo degli incendi immani, due corrazzate russe e un'inglese. Più tardi giunsero le corrazzate italiane. Nel nostro quartiere, si diedero a un'opera di eroico salvataggio i marinai russi, ammirevoli e ammirati da tutti come fratelli e salvatori (Bruschettini, 2009).
\end{abstract}

I fatti narrati dal prof. Bruschettini si svolsero tra il 28 e il 29 dicembre 1908 e testimoniano la presenza delle navi russe, tra le prime a portare soccorso alla città di Messina. II terremoto di Messina colse impreparate le autorità regie. Le molteplici istituzioni, la Prefettura, i comandi militari e le autorità statali mancarono di rapidità nell'organizzare i primi necessari soccorsi. Inoltre tutte le linee di comunicazione saltarono, le prime notizie sul tragico evento furono inviate nel pomeriggio dalle superstiti autorità militari. A Reggio i primi soccorsi furono organizzati dal generale Achille Mazzitelli in uno scenario apocalittico, a Messina regnava la confusione, tutte le caserme erano distrutte. Fu il rapido intervento di alcune unità straniere a fornire un primo soccorso alle aree devastate dal sisma, le navi russe. Al comando dell'ammiraglio Litvinov la squadra dell'armata navale imperiale russa, formata dalle corazzate Slava e Cesarevic, dagli incrociatori Makarov e Bogatyr' e dalle cannoniere Giljak e Koreec. Le navi russe, impegnate in quei giorni in un'esercitazione nel Mediterraneo occidentale, il 28 dicembre si trovavano nel porto di Augusta, a circa $140 \mathrm{~km}$ da Messina, dove si sparse la notizia del terremoto. L'ammiraglio Litvinov, secondo quanto riportarono le cronache del tempo, convocò un consiglio per organizzare con gli ufficiali un'operazione di primo soccorso, salpando in direzione porto di Messina. Viste le molteplici difficoltà di manovra, i detriti in mare e la confusione generale, i marinai russi calarono numerose scialuppe con a bordo unità di soccorso, molti raggiunsero la riva a nuoto, tra cadaveri, assi di legno e ogni sorta di orribile segno della devastazione appena passata. I russi s'impegnarono nella ricerca dei superstiti sotto le macerie, tra le montagne di detriti, trasportarono i feriti a bordo delle proprie unità e distribuirono cibo ai numerosi sopravvissuti. Intanto, sulle banchine della Marina, alcune sezioni della Sanità russa, coadiuvate dal capo chirurgo della squadra Alexander Bunge, allestirono un provvisorio ospedale da campo. Scrisse il politico e giornalista Claudio Treves, 
sul Tempo il 4 gennaio 1909, in riferimento ai marinai russi: "Meravigliano i soldati russi che fanno prodigi di valore; a essi spettano i primi onori, oltreché per la grande abnegazione, per il metodo, la precisione, la freddezza del loro lavoro" (Mercadante, 2009), evidenziò Giuseppe Piazza, giornalista, sulla Tribuna il 2 gennaio 1909: "Unica nota di conforto, fra tanto orrore, sono i veri prodigi di valore operati dagli equipaggi delle due navi russe Bogatyr' e Slava" (Mercadante, 2009), esaltò lo scrittore Giovanni Cena sulla Nuova Antologia il 31 gennaio 1909: "Finora la storia ha celebrato eroismi individuali, eroismi di singole città e nazioni. Per la prima volta nel mondo, dei soldati, recanti la divisa degli antagonismi nazionali, si sono sentiti uomini di fronte alla sventura di altri uomini. Marinai russi e inglesi, prima, poi di tutte le nazioni, hanno gareggiato nella carità" (Mercadante, 2009) volgendo l'attenzione sull'intricato assetto politico internazionale, ricco di antagonismi e gelosie. Eroica la descrizione di Carlo Antonio Piazza, l'8 gennaio 1909, sul Corriere d'Italia: "Fuori dal molo dondola leggermente, nella sua enorme mole olivastra, la corazzata russa, Slava. II colossale alveare d'acciaio, irto di cannoni e superbo di bellezza, riposa in un silenzio alto di monastero. I grossi marinai, dagli occhi dolci come di fanciulli, sono discesi a terra, la piatta pala cosacca si confonde col piccone della nostra fanteria. Che bravi ragazzi! lo li osservo da tre giorni ai piedi d'una casa diroccata e non ho mai scorto nel loro sguardo un lampo di ripugnanza, né nelle loro braccia un segno di stanchezza [...]" (Mercadante, 2009).

Matilde Serao, scrittrice e autrice di numerose opere, giornalista, è stata la prima donna in Italia ad aver fondato e diretto un quotidiano, seguì la tragedia del terremoto e scrisse su I/ Giorno importanti memorie, soprattutto l'articolo intitolato Marinai russi:

\begin{abstract}
Da ventiquattrore, in uno scenario terrificante di rovine, fra le fiamme dell'incendio, in Messina, gemevano sotto le pietre i sepolti vivi e a un tratto sono apparsi dei visi umani, contratti dalla sorpresa, dall'ansietà e dalla pietà: degli uomini, sono apparsi, venendo dal mare, scendendo da una nave, avanzandosi fra le macerie, scavalcando montagne di pietre e di calcinacci. Erano naviganti, ufficiali e marinai: di un'altra nazione, giunti da mari lontani, da mari nordici, appartenenti a una nave da guerra, alla nave russa, l'Admiral Makharoff. E questi pochi, ufficiali e marinai, si sono messi a estrarre i sepolti vivi da sotto le pietre delle case di Messina, essi per primi: si sono messi a raccogliere feriti, a cercare di medicarli, di sollevarli, con qualche cordiale, essi per primi, questi russi, dal nobilissimo loro comandante al più oscuro dei mozzi. Cosi, molto, molto più che l'Admiral Makharoff ne potesse imbarcare, sono stati imbarcati i feriti, tanta era ardente la volontà di salvezza di questi russi (Mercadante, 2009).
\end{abstract}

Ancora una volta, nel vivo racconto della Serao, scorgiamo il senso umanitario, profondo, dei marinai russi, la loro abnegazione e l'eroismo delle loro gesta, dal primo all'ultimo degli uomini dell'equipaggio, dagli alti ufficiali ai semplici mozzi, tutti scavarono fra le macerie, tutti aiutarono, come poterono, qualsiasi uomo, donna e bambino che incontrarono sul loro cammino, in un contesto di totale distruzione e angoscia che solo un terremoto può lasciare. Altro compito svolto dai marinai russi era stato quello di ristabilire ordine e sicurezza, evitando così il triste fenomeno dello sciacallaggio. Atti di eroismo quindi, così definì la comunità messinese il sacrificio dei primi soccorritori russi, che già nel primo consiglio comunale, successivo al sisma, aveva deliberato l'erezione di un monumento ai russi che, proseguendo nella solidarietà, avevano fondato finanche un comitato Pietroburgo - Messina per raccogliere fondi per la ricostruzione, lo stesso zar aveva deciso di donare 50000 franchi alla città siciliana (Dickie, 2008). In seguito, ai primi soccorsi della flotta dello zar Nicola II, erano intervenute navi inglesi, francesi e americane (Motta, 2008), complessivamente i russi salvarono 800 persone e prestarono soccorso a più di 2500 vittime del disastro (Motta, 2008). Dopo i primi soccorsi internazionali, tra le polemiche, arrivarono le Forze Armate italiane e fu designato un commissario straordinario per i circondari di Messina e Reggio Calabria, il tenente generale Francesco Mazza. Una settimana dopo il terremoto, ricorda Alessandro Vagnini nel suo Le navi, simbolo degli aiuti internazionali, erano circa settemila i marinai italiani impegnati nell'opera di soccorso e conclude evidenziando l'impressionante contributo fornito dalle navi italiane e straniere, mobilitazione testimonianza "della partecipazione del mondo a quella disgrazia naturale" (Motta, 2008), anche perché il terremoto di Messina assumeva rilevanza anche per ciò che riguardava l'aspetto politico internazionale.

\title{
3. Politica internazionale e considerazioni finali
}

E' Antonello Biagini a ricordarci nel suo saggio Linee di politica europea all'interno del volume curato da Giovanna Motta La città ferita, che il 1908, l'anno balcanico per eccellenza (Motta, 2008), vide la prima importante crisi all'interno della Triplice Alleanza (tra Impero austro-ungarico, Impero tedesco e Regno d'Italia, 1882) a causa del contrasto tra Impero austro-ungarico e Regno d'Italia generato dall'annessione della Bosnia da parte austriaca, in totale inosservanza di quanto previsto nel trattato che aveva fissato il principio dell'informazione preventiva e quello dei compensi in caso di 
modifiche dello statu quo nei Balcani (Sciarrone, 2013). II trattato della Triplice, per l'Italia, comportava una posizione contraria all'Inghilterra, visti i rapporti positivi si era deciso di aggiungere una dichiarazione che specificasse che l'accordo non era in funzione anti inglese (Giordano, 2008). Quindi, da quel momento in poi e sino allo scoppio della prima guerra mondiale, l'Italia cercò di rispettare i vincoli del trattato della Triplice Alleanza (Giordano, 2004). Dallo studio della documentazione conservata presso gli Archivi italiani, la politica estera italiana a partire dal XIX secolo ha cercato di raggiungere visibilità in campo internazionale attraverso la partecipazione attiva ai corpi di spedizione multinazionali. Nel periodo qui studiato i rapporti tra le grandi potenze si aggravano, la Triplice non si dimostra disponibile a sostenere le mire italiane in Africa e non vede positivamente le amicizie tra Italia, Francia e Inghilterra, Visconti Venosta avrebbe voluto impedire nei Balcani, il "direttorio" a due, avviato da Vienna e Pietroburgo ma la crisi bosniaca del 1908 rende inutile tale pianificazione poiché l'annessione da parte dell'Austria avviene con il consenso della Russia. Una lunga trattativa porterà, tramite il ministro degli esteri Tommaso Tittoni, all'incontro di Racconigi, 23 ottobre 1909, in cui Italia e Russia si accordarono per garantire i due Paesi in caso di conflitto del primo con l'Austria e del secondo con l'Inghilterra e Austria. II terremoto di Messina si pone quindi in un periodo delicato e difficile degli equilibri internazionali e funziona, secondo Biagini, come "altro elemento di contrapposizione malgrado prevalga un coinvolgimento mondiale che si avvale di molte e diverse energie" (Motta, 2008). Resterà pertanto scolpita nella storia e nella memoria l'enorme pagina di eroismo e sacrificio della marina russa, impeto di solidarietà che coinvolge, per la prima volta, ogni Paese e che distrugge decenni di rivalità e contrapposizioni politiche, come scrisse il grande scrittore Gor'kij: "Il dolore di Messina è il dolore del mondo" (Motta, 2008).

\section{Bibliography}

Consolo Langher S.N. (2005), Espansionismo greco e rivendicazioni sicule: guerra e pace nei secoli VI e V a. C., cit. in Calogero Miccichè, Simona Modeo, Luigi Santagati ( a cura di ), Diodoro Siculo e la Sicilia indigena, Caltanissetta, Atti del Convegno di studi su Diodoro Siculo e la Sicilia indigena

Dickie V.J. (2008), Una catastrofe patriottica, 1908: il terremoto di Messina, Roma - Bari, Laterza

Frisina T. (2008), Il Terremoto di Reggio e Messina del 28 dicembre 1908 nel centenario dell'evento - cartoline e cronache d'epoca, Castelnuovo Scrivia, II Mosaico Kids

Giordano G. (2008), Cilindri e feluche, La politica estera dell'Italia dopo l'Unità, Roma, Aracne editrice

Giordano G. (2004), Storia della politica internazionale 1870 - 2001, Milano, FrancoAngeli

Mercadante F. (2009), II terremoto di Messina. Corrispondenze, testimonianze e polemiche giornalistiche, Reggio Calabria, Città del Sole Edizioni

Motta G. (1998), I turchi, il Mediterraneo, l'Europa ( a cura di), Milano, FrancoAngeli

Motta G. (2008), La città ferita, II terremoto dello Stretto e la comunità internazionale, Milano, Franco Angeli

Ribot L. (2011), La rivolta antispagnola di Messina, Catanzaro, Rubbettino Editore

Sciarrone R. (2013), Strategie militari franco-tedesche a confronto, 1905-1913, Roma, Edizioni Nuova Cultura 